Lingüística

Vol. 33-2, diciembre 2017: 33-53

ISSN 2079-312X en línea

DOI: $10.5935 / 2079-312 X .20170016$

\title{
ELISIÓN DE LA RAÍZ VERBAL
}

\author{
VERB ROOT ELLIPSIS
}

\author{
Bernard Comrie \\ University of California, Santa Barbara \\ comrie@linguistics.ucsb.edu \\ Raoul Zamponi \\ Investigador independiente \\ zamponi_raoul@libero.it
}

\section{Resumen}

La elisión de la raíz verbal es un fenómeno atestiguado inequívocamente solo en dos lenguas habladas en América y una familia lingüística de las Islas Andamán. Consiste en la elisión de la raíz del verbo, en primer lugar bajo condiciones de recuperabilidad, dejando solamente afijos (y eventualmente clíticos) y, de esta manera, sometiendo a prueba los límites de la teoría y de las posibilidades morfológicas. El fenómeno es aquí examinado en las lenguas pertinentes: inuktitut, kwaza y algunos miembros de la familia gran andamanesa. Se discuten paralelos formales y funcionales con otros fenómenos tales como la elisión del verbo (elisión de la palabra entera) y morfemas y alomorfos de raíces léxicas cero.

Palabras clave: morfología, elisión, raíz cero

Verb Root Ellipsis is a phenomenon attested robustly in only two languages spoken in the Americas and one language family of the Andaman Islands. It consists in the ellipsis of the root of the verb, primarily under conditions of discourse retrievability, leaving behind only affixes (and possibly clitics), thus testing the bounds of morphological possibilities and theory. The phenomenon is examined in the relevant languages: Inuktitut, Kwaza, and some members of the Great Andamanese family. Formal and functional parallels with other phenomena, such as verb ellipsis (ellipsis of the whole verb word) and lexical zero-root morphemes and allomorphs are discussed.

Keywords: morphology, ellipsis, zero root 
Recibido: $04 / 06 / 2017$

Aceptado: 28/07/2017

\section{Introducción}

Partiendo de sus experiencias en el análisis del lenguaje y de las teorías que aspiran a generalizar esas experiencias, los lingüistas tienen a menudo intuiciones muy claras sobre la posibilidad o la probabilidad de varios fenómenos en el lenguaje humano. Una de estas intuiciones concierne a los tipos de morfemas que pueden ocurrir como morfemas cero, como en la formulación siguiente de Trommer (2012: 353):

It is a common intuition that $\varnothing$-morphemes are only possible for very specific types of morphemes. They are expected to occur as affixes, but not as roots. Moreover, $\varnothing$ seems more likely with functional material (whether affixal or not) than with lexical morphemes.

La formulación de Trommer es muy cautelosa en términos de probabilidades e improbabilidades y no de posibilidades e imposibilidades. En este artículo se examina un tipo de excepciones a su generalización, a saber la elisión de la raíz verbal.

Bajo el término "elisión de la raíz verbal" nos referimos a una palabra con una raíz que generalmente es expresada abiertamente pero puede también estar ausente, con retención de los demás morfemas de la palabra, bajo determinadas condiciones, sobre todo discursivas. El mini-diálogo en (1) ejemplifica este fenómeno en lengua kwaza (véase la sección 2.2 para más detalles).

1. a. ku'ro-xa-xa-re

cerrar-2S-PA-INT

'¿Cerrasteis [la puerta]?'

b. Ø-'a-xa-ki

RE-1PLS-PA-DEC

'La [cerramos].' (van der Voort 2004: 579)

La raíz verbal ku'ro- 'cerrar' está expresada abiertamente en el primer turno (a) del diálogo, pero falta en el segundo turno (b), que consiste en una pura serie de sufijos.

El fenómeno es inesperado según la intuición formulada por Trommer y, que sepamos, se encuentra solamente en tres casos independientes: inuktitut, kwaza y algunas lenguas de la familia gran andamanesa. ${ }^{1}$

Nuestro objetivo en este artículo es proponer un análisis en términos de elisión de la raíz verbal, aunque nuestra discusión y, sobre todo, su dimensión

\footnotetext{
${ }^{1}$ En Comrie y Zamponi (en prep.) consideramos un cuarto caso, la lengua australiana jingulu, concluyendo que no se trata de un caso inequívoco de elisión de la raíz verbal.
} 
tipológica es necesariamente preliminar, ya que son poquísimas las lenguas que manifiesten este fenómeno.

En la sección 2 examinamos en detalle el fenómeno de la elisión de la raíz verbal en los tres casos antedichos. La sección 3 trata las consecuencias fonológicas de la elisión de la raíz, mientras que en la sección 4 evidenciamos semejanzas y diferencias entre la elisión de la raíz verbal y otros dos fenómenos, a saber la elisión verbal (vinculada funcionalmente a la elisión de la raíz verbal) y las raíces cero (vinculadas a la elisión de la raíz verbal no solo formalmente, debido a la ausencia de una raíz expresada abiertamente, sino también semánticamente, teniendo en cuenta la semántica de las raíces cero y de las raíces elididas fuera de condiciones puramente discursivas).

\section{Elisión de la raíz verbal en inuktitut, kwaza y akabea}

En el estado actual de nuestros conocimientos, son poquísimas las lenguas en las cuales la elisión de la raíz de un verbo es permitida: el inuktitut (2.1) y el kwaza (2.2) en América; el akabea y otras dos lenguas en las islas Andamán (2.3).

\subsection{Inuktitut}

El inuktitut es un conjunto de variedades dialectales habladas en el Ártico oriental canadiense aproximadamente por 36.000 personas. Su agrupación lingüística forma parte de la rama esquimal de la familia esquimo-aleutiana. Debe tenerse en cuenta que el fenómeno de la elisión de la raíz verbal interesa específicamente al dialecto tarramiut del norte del Quebec (bahía de Ungava y estrecho de Hudson) y otras variedades canadienses orientales, pero no a los restantes dialectos inuktitut, y que el mismo fenómeno parece ser ajeno ya a los dialectos inuit de Groenlandia, estrechamente relacionados, ya a las más lejanas variedades yupik del extremo oriental de Siberia y Alaska. La breve discusión que sigue se basa en la descripción del fenómeno en la variedad tarramiut del inuktitut canadiense oriental [ike] hablada en el estrecho de Hudson ofrecida por Swift y Allen (2002). La información para este trabajo fue a su vez sacada de un corpus constituido por material recolectado en un proyecto sobre la adquisición del inuktitut por parte de niños en el que se destaca también una amplia muestra de oraciones de adultos dirigidas a niños $\mathrm{u}$ otros adultos en el ámbito de actividades de cuidado infantil. Como los autores del trabajo indican (p. 133), la elisión de la raíz verbal no está, sin embargo, confinada a tales contextos conversacionales, siendo también recurrente en los diálogos entre adultos.

Como en otros dialectos y lenguas esquimales, la estructura mínima de un verbo en el inuktitut tarramiut consiste en una base seguida por un sufijo flexivo a los cuales pueden agregarse una o más posbases y partículas enclíticas en las posiciones indicadas en el esquema siguiente.

2. BASE $(+$ POSBASES $)+$ SUFIJO FLEXIVO (+ ENCLÍTICOS $)$ 
La base, por otra parte, puede consistir en una raíz o una raíz sufijada por determinados morfemas derivacionales. Las posbases pueden ya cambiar la base de una a otra clase sintáctica (como, por ej., el verbalizador -it-), ya marcar tiempo, aspecto o modo del verbo. Los sufijos flexivos que siguen a la base de un verbo son marcas de modo $y$, en las formas intransitivas, del número y persona del sujeto 0 , en las formas transitivas, del sujeto y del objeto.

Para ser exacto, lo que se puede elidir en un verbo inuktitut es su base y no solamente la raíz, en presencia de otros morfemas. Ya que, sin embargo, la elisión afecta siempre y obligatoriamente a la raíz del verbo, seguiremos usando el término "elisión de la raíz verbal" para referirnos al fenómeno en cuestión. ${ }^{2}$ Cabe también señalar que algunas posbases (como, p. ej., el verbalizador -it-) excluyen la posibilidad de elidir la raíz/base de un verbo.

Exceptuando posibles casos de expresiones convencionalizadas, la elisión de una raíz verbal es posible solo cuando la raíz puede ser recuperada por el contexto $y$, típicamente, este no es más que un turno conversacional anterior, como en (3) y (4). En (3a), el primer hablante introduce la noción de jugar. En $(3 b)$, la primera palabra de la respuesta elide este contenido y, sobre un plano estrechamente formal, consiste exclusivamente en un sufijo flexivo que marca simultáneamente modo dubitativo (el cual permite formular el equivalente de una pregunta indirecta del español) y sujeto de tercera persona plural.

3. a. Aala atsakuttit pinnguatui maani ${ }^{3}$

aala atsa-kkut-tit pinnguaq-juq-it ma-ani

escucha tía_materna-PLAS-ABS.2SG>PL jugar-NOMZ-ABS.PL aquí-LOC

'Escucha, tu tía y su amigos/amigas están jugando aquí'

b. Mangata takulaukkinai

$\varnothing$-mmangata taku-lauq-kkit=ai

BE-DUB.3PL ver-COR-IMP.2SGS>3PL=ENF

'Por favor, mira si [están jugando].' (Swift y Allen 2002: 150)

Del mismo modo, la oración en (4b) introduce una noción no expresada abiertamente en el turno conversacional siguiente: la llegada de Tracy.

4. a. Traci qaiju

Traci- $\varnothing \quad$ qai-juq

Tracy-ABS:SG venir-PAR.3SGS

'Tracy está viniendo.'

b. Quurmat

$\varnothing$-qquuq-mmat

BE-probablemente-cTG.3sGS

Probablemente [está viniendo].' (Swift y Allen 2002: 147)

\footnotetext{
${ }^{2}$ Téngase, sin embargo, en cuenta que, en los ejemplos contenidos en esta sección, en vez de poner la glosa RE para lo que viene elidido, usaremos la glosa BE.

${ }^{3}$ A la transcripción de los ejemplos de esta sección según la ortografía estándar del inuktitut siguen su análisis morfológico, las glosas de los morfemas y la traducción, exactamente como en Swift y Allen (2002).
} 
Es también posible que la raíz verbal omitida sea recuperable del contexto situacional concreto, como en (5) y (6). El ejemplo (5) retoma lo que la madre dice a la pequeña Louisa (que está llorando) después de haberle llevado, por un rato, su almohada. Esto es, simplemente, un verbo formado por una posbase que codifica un tiempo verbal $y$ un sufijo flexivo que indica simultáneamente modo, persona y número del sujeto y del objeto sin alguna raíz que vehicule explícitamente el significado lexical 'volver a tener'.

5. (por parte de la madre a Louisa que exige su almohada)

Langagaviuk

$\varnothing$-langa-gaviuk

BE-FUTPROX-CTG. 2SGS>3SGO

'[Volverás a tener]la pronto.' (Swift y Allen 2002: 144) puzzle.

El ejemplo (6) retoma lo dicho por Louisa al añadir la última pieza de un

\section{Giirpunga}

$\varnothing$-jariiq-vunga

BE-TERM-IND.1SGS

'Terminé (de componer el puzzle).' (Swift y Allen 2002: 145) ${ }^{4}$

El ejemplo (7) podría igualmente ser analizado teniendo en cuenta el contexto extralingüístico: contexto que seguramente deja en claro lo que el hablante quiere hacer y pide poder hacer. El ejemplo, en concreto, muestra una forma verbal en la que podemos reconocer una posbase, un sufijo flexivo y un enclítico, pero no una raíz. Swift y Allen señalan que esta enunciación parece ser el equivalente convencionalizado de la expresión inglesa my turn 'mi turno' (o 'Me toca a mí') y esto deja abierta la posibilidad de que, aunque tenga la estructura de un verbo con raíz/base elidida, la construcción en (7) sea considerada una forma lexical idiomática autónoma.

\section{Laurlangali \\ $\varnothing$-lauq-langa $=$ li \\ BE-COR-IMP. 1 SGS = and}

'Me toca a mí' (lit. 'Por favor déjame'). (Swift y Allen 2002: 136)

Las pocas construcciones elípticas aquí presentadas, o por lo menos las no convencionalizadas en (3-6), evidencian una condición indispensable por la elisión de la raíz de un verbo en inuktitut que es la recuperabilidad del material elidido a partir del contexto, sea este lingüístico (como lo es un turno conversacional precedente) o puramente situacional.

\footnotetext{
${ }^{4}$ La primera sílaba de la posbase -jariiq- tiende a caer cuando sigue una base plena y es siempre abreviada en las construcciones elípticas.
} 


\subsection{Kwaza}

Otra lengua nativa americana que conoce el fenómeno de la elisión de la raíz verbal es el kwaza (o koaia) [xwa], idioma no clasificado hablado en el estado brasileño de Rondonia por apenas siete personas (dato del 2010). La lengua fue descripta por Hein van der Voort en una amplia y detallada gramática (van der Voort 2004) y la presente sección de este artículo se basa casi exclusivamente en esta obra.

Hay que tener presente que los verbos del kwaza empiezan todos con una raíz la cual puede ser seguida por un cierto número de sufijos entre los cuales figuran obligatoriamente una marca de persona y una de modo. El fenómeno de elisión de la raíz verbal en esta lengua, a diferencia del inuktitut, implica precisamente la supresión de la sola raíz del verbo y el mantenimiento de todos los sufijos, en particular de los obligatorios que marcan la persona del sujeto y el modo.

Van der Voort (p. 590) indica que algunos verbos de habla del kwaza podrían reflejar una lexicalización de la elisión de la raíz verbal. En esta sección haremos referencia solo a casos de elisión de la raíz verbal productiva y no nos ocuparemos de tales verbos.

Van der Voort (pp. 578-579) hace también notar que la elisión de la raíz verbal es particularmente frecuente en los diálogos $y$, específicamente, en construcciones que corresponden a respuestas mínimas a preguntas, comentarios y observaciones. Los mini-diálogos en (1) y (8) son ejemplos de esta situación. La raíz omitida del verbo en $(1 b)$ es $k u$ 'ro- 'cerrar', recuperable de la pregunta inmediatamente anterior (1a), como hemos dicho en 1 . Del mismo modo, es posible recuperar la raíz elidida del verbo (8b), o'ja- 'irse', gracias a la pregunta precedente que se observa en (8a).

8. a. o'ja-xa-tsy-re

irse-2S-POT-INT

'¿Estás yendo?'

b. $\varnothing$-'da-tsy-tse

RE-1SGS-POT-DEC

'Me [voy].' (van der Voort 2004: 579)

Otros ejemplos de respuestas elípticas aparecen a continuación en (9c) y $(10 c)$, en paralelo con sus versiones no elípticas en $(9 b)$ y $(10 b) .{ }^{5}$

Las raíces omitidas en (9c) y (10c) son, respectivamente, ja- 'comer' y kui- 'beber, tomar', observables en las oraciones en (9a) y (10a).

9. a. ja-'e-da-mỹ

comer-de_nuevo-1SGS-VOL

'Voy a comer de nuevo.'

b. ja-e-'ra

comer-de_nuevo-Imp

'iCome de nuevo!'

${ }^{5}$ Nótese, sin embargo, la presencia del sufijo -je?e 'de nuevo' en (10c), no utilizado en (10b). 


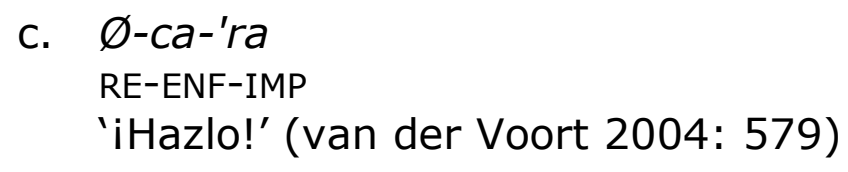

10. a. kui-'nã-xa-re

tomar-FUT-2S-INT

'¿Tomarás?' (van der Voort 2004: 580)

b. kui-'he-je?e-nã-da-ki

tomar-NEG-again-FUT-1SGS-DEC

'No tomaré más/de nuevo.' (van der Voort 2004: 533)

C c. Ø-'he-nã-da-ki

RE-NEG-FUT-1SGS-DEC

'No lo haré.' (van der Voort 2004: 580)

Como en inuktitut, la interpretación de las construcciones elípticas sin raíz verbal puede también depender completamente del contexto situacional en kwaza. Un ejemplo de esto (correspondiente a la primera parte del turno conversacional (64) en van der Voort $(2004: 763,766)$ ) nos lo señala van der Voort (comunicación personal).

11. $\varnothing$-'da-ta'ra-tse

RE-1SGS-POSP-DEC

'Me [voy].'

El contexto situacional en el cual este ejemplo se coloca muestra al hijo decir a su madre que la entrevista está terminada para aquel día y que otra tendrá lugar el día siguiente. La madre pronuncia el verbo elíptico en (11) y sigue diciendo que, en aquel momento, hace un calor insoportable y que el próximo día será posible trabajar de nuevo. Nada en el contexto lingüístico sugiere que el contenido semántico de la raíz omitida es 'ir'.

El kwaza presenta una manifestación prototípica del fenómeno de la elisión de la raíz verbal. Es exactamente la raíz del verbo que viene a ser elidida y esto es posible cuando dicha raíz es recuperable en el contexto lingüístico previo o en el contexto situacional extralingüístico.

\subsection{Akabea (y otras lenguas gran andamanesas)}

También en akabea la elisión de la raíz no interesa más que a la raíz de un verbo pero, a diferencia de lo que ocurre en inuktitut y en kwaza, esta elisión puede resultar no relacionada ni con el contexto lingüístico previo ni con el contexto extralingüístico.

El akabea [abj] es una lengua extinta entre 1921 y 1931, anteriormente hablada en el archipiélago de Andamán $y$, exactamente, en las islas de Andamán del Medio y Andamán del Sur y otras menores próximas a Andamán del Sur. Pertenece a la familia lingüística gran andamanesa, un grupo de siete lenguas cercanamente emparentadas ( $\sin$ relaciones externas conocidas) que fueron habladas en Gran Andamán (las cinco islas principales del norte de las 
Andamán) e islas e islotes cercanos. De hecho, también la familia gran andamanesa entera se halla extinguida dado que lo que resta hoy de esta son solo cinco hablantes parciales (con varios niveles de competencia) de una amalgama de los viejos dialectos de la isla de Andamán del Norte (akajeru, akabo, akakhora y akachari) Ilamada "gran andamanés contemporáneo" (Present-day Great Andamanese) o, simplemente, "gran andamanés".

Conocemos el akabea y las otras lenguas gran andamanesas tradicionales, que dejaron de ser habladas durante la primera mitad del siglo veinte, a través, sobre todo, de un "manual" de akabea, opuchikwar, akakede y akachari provisto de un vocabulario comparativo y un fraseario (Portman 1887), de una obra que reúne notas gramaticales, material léxico y textos del akabea, del akarbale, del opuchikwar, del okojuwoi y del okol (Portman 1898), de un diccionario inglés-akabea (Man 1919-1923), de una gramática inédita del akabea que se halla incompleta (Man 1878) y de una importante monografía sobre las sociedades andamanesas de Alfred R. Radcliffe-Brown que contiene, passim, inserciones (palabras, sintagmas y oraciones) de varias lenguas del archipiélago andamanés (Radcliffe-Brown 1933). Del gran andamanés contemporáneo disponemos de varios trabajos, entre ellos, una gramática de referencia (Abbi 2012) y un diccionario (Abbi 2013).

La posibilidad de elidir la raíz de un verbo es documentada, además del akabea, en otras dos lenguas de la familia gran andamanesa y, precisamente, el opuchikwar [apq] (Baratang y Andamán del Medio) y el okojuwoi [okj] (Andamán del Medio). Las descripciones disponibles del gran andamanés contemporáneo no mencionan este fenómeno y tampoco hay rastros de esto en la documentación disponible del akachari, dialecto que constituye parte de la herencia lingüística de los últimos hablantes (o "rememberers") del gran andamanés contemporáneo.

No sabemos si esto es debido a una efectiva ausencia del fenómeno en gran andamanés contemporáneo y akachari o, simplemente, en el material de estas variedades que fue recogido. La más amplia variedad de construcciones con raíz verbal elidida se encuentra, por supuesto, en akabea, la lengua gran andamanés tradicional mejor documentada. Una típica estructura verbal del akabea es la siguiente.

\section{PRONOMBRE CLÍTICO + PREFIJO_SOMÁTICO (+ NÚMERO) + RAÍZZ + TAM ${ }^{6}$}

Precisamente son tres o, quizá, cuatro los tipos productivos de elisión de la raíz verbal que se observan en el corpus del akabea (Zamponi y Comrie en prep.: Secc. 3.3).

Uno de estos tipos consiste en la elisión de una raíz verbal recuperable de un turno conversacional anterior como, por ejemplo, cuando el verbo ocurre en una orden (véase (13)) y resulta hallarse, por lo tanto, implícito en la respuesta (veáse (14)). ${ }^{7}$

\footnotetext{
${ }^{6}$ La marca de número (plural) precede el prefijo somático cuando este último es ig- idz-.

${ }^{7}$ En la primera línea de los ejemplos de esta sección se observa su representación ortográfica original. En la segunda línea hemos incluido nuestra transcripción semi-fonética así como su análisis morfémico.

Siguen, como siempre, las glosas morfémicas y la traducción española.
} 
13. Âchitik [sic] reg dama màgke

$\begin{array}{llll}\text { atfitek } & \text { reg } & \text { dama } & \text { mek-ke } \\ \text { ahora } & \text { puerco } & \text { carne } & \text { comer-nPSD }\end{array}$

'iComerás carne de puerco ahora!' (Man 1878: Cap. 34)

14. Yabada, wai dîlalen dôke
yaba=da / wai
dila $=$ len
d-o
$\varnothing-k e$
NEG $=$ COP
FOC
tarde $=$ LOC 1 SG-S.SG
RE-nPSD
'No (lit. No es). [La comeré] en la tarde.' (Man 1878: Cap. 34)

Otro tipo de elisión es la de la raíz del verbo de movimiento on 'venir, ir' en el caso de que esta sea clara en el contexto intraoracional, típicamente porque hay un adverbio o un sintagma posposicional que indica dirección y un sujeto animado. ${ }^{8}$

15. Israel ér-len kátik-ké

$\begin{array}{llll}\text { Israel } & \text { er=len } & \text { katik } & \varnothing \text {-ke } \\ \text { Israel } & \text { tierra=LOC } & \text { allá.ESP } & \text { RE-nPSD }\end{array}$

'En tierra de Israel, i [irás] allá!' (Portman 1898: 158)

16. Dó yát taijnga látké

$\begin{array}{llll}\text { d-o } & \text { yat } & \text { taidz-na=lat } & \emptyset-k e \\ \text { 1SG-S.SG } & \text { pez } & \text { pegar_un_tiro-NOMZ=AL } & \text { RE-nPSD } \\ \text { 'Yo me [voy] a pescar.' (Portman 1887: 146) } & \end{array}$

17. ... á 'en-iji-múg-éni-nga l'át-ré

$\varnothing$-a en-idz-i-mug-eni-na=lat $\varnothing$-re

3-O PREV-PS-REFL-frente-agarrar-NOMZ $=$ AL $\quad$ RE-PRET

'[Vinimos] a adorarlo.' (Portman 1898: 134, 136)

18. Ka wai dôke

$\begin{array}{llll}\text { ka } & \text { wai } & \text { d-o } & \varnothing-k e \\ \text { DEM.PROX } & \text { FOC } & \text { 1SG-S } & \text { RE-nPSD } \\ \text { 'iMe [voy]!' (Man 1878: Cap. 9) } & \end{array}$

Esto tipo de elisión de la raíz verbal puede también ser observado en el escaso material opuchikwar y okojuwoi a nuestra disposición.

19. ... uk taiye péne láteke
$\varnothing$-uk
taiye
pene $=$ late
$\varnothing-k e$
3SG-S pez agarrar $=\mathrm{AL}$ RE-nPSD
'Él [va] a pescar.' (Portman 1887: 146)

20. ... á Egypt láte-chíkan
$\varnothing-a$
Egypt=late
$\varnothing$-kitfikan
3SG-S.SG
Egipto $=A L$
RE-PRET
'Él (José) [fue] a Egipto.' (Portman 1898: 151)

(opuchikwar)

(okojuwoi)

\footnotetext{
${ }^{8}$ En (18) no hay una indicación de dirección.
} 
En su gramática inédita del akabea, Edward H. Man proporciona varios ejemplos de elisión de la raíz verbal pere 'golpear (con un palo o un arma)' (21b-24). Todos estos ejemplos, excepto el de (23), incluyen un agente humano o inanimado y un paciente humano expresados abiertamente. La oración en (23), en cambio, contiene solamente un paciente expresado de modo manifiesto. Los ejemplos (21a) y (21b) muestran versiones de una misma oración con y sin raíz verbal respectivamente. A pesar de la ausencia de la raíz, en las formas verbales en $(21 b-24)$ se observa la retención de un prefijo somático, que señala la parte del cuerpo golpeada, y de una marca temporal sufijal. Los mismos ejemplos contienen también un pronombre personal objeto proclítico que se apoya sobre la forma verbal sin raíz. El ejemplo (23a) presenta además una marca de plural entre pronombre personal objeto proclítico y el prefijo somático.

21. a. Dô ngôtpàreke

$$
\begin{array}{ll}
\text { d-o } & \eta=\text { ot-pere-ke } \\
\text { 1SG-S.SG } & \text { 2O=PS-golpear-nPSD }
\end{array}
$$

'Yo te golpearé en la cabeza.' (Man 1878: Cap. 34)

b. ... do ngôtke

$$
\begin{array}{ll}
\text { d-o } & \eta=\text { ot- } \varnothing-k e \\
1 \text { SG-S.SG } & 2 O=\text { PS-RE-nPSD } \\
\text { 'Yo te [golpearé] en la cabeza.' (Man 1878: Cap. 34) }
\end{array}
$$

22. Kâto êla dôngre

$$
\begin{array}{lll}
\text { kato } & \text { ela } & \mathrm{d}=\text { on- } \varnothing \text {-re } \\
\text { DEM.DIST } & \text { flecha_para_matar_a_los_puercos } & 1 \mathrm{SGO}=\text { PS-RE-PRET }
\end{array}
$$

'Aquella flecha me [golpeó] en la mano/el pie.' (Man 1878: Cap. 34)

23. a. Michîba ngitigre?

mitfiba $\quad \eta=i t-i g-\varnothing-r e$

qué $\quad 2 \mathrm{O}=$ PL-PS-RE-PRET

'¿Qué te/os [golpeó] en los ojos?' (Man 1878: Cap. 35)

b. Michîba ngabre

mitfiba $\quad \eta=a b-\varnothing-r e$

qué $\quad 2 \mathrm{O}=\mathrm{PS}-\mathrm{RE}-\mathrm{PRET}$

'¿Qué te/os [golpeó] en el cuerpo?' (Man 1878: Cap. 35)

c. Michîba ngâkâre

$$
\begin{array}{ll}
\text { miţiba } & \eta=a k a-\varnothing-r e \\
\text { qué } & \text { 2O=PS-RE-PRET } \\
\text { '¿Qué te/os [golpeó] en la boca?' (Man 1878: Cap. 35) }
\end{array}
$$

24. Kichîkachâ ngôngre?

$$
\begin{array}{ll}
\text { kitfikatfa } & \eta=\text { on- } \varnothing \text {-re } \\
\text { cómo } & \text { 2O=PS-RE-PRET } \\
\text { '¿Cómo te/os [golpeó] en la mano/el pie?' (Man 1878: Cap. 34) }
\end{array}
$$


Una oración registrada por Maurice V. Portman (véase ejemplo (25)) deja suponer la presencia en akabea de un cuarto tipo de elisión de la raíz verbal representado por la omisión de la raíz del verbo copulativo eda $\sim=d a$ 'ser' no condicionada por factores gramaticales específicos. Esta oración consiste en una cláusula verbal negativa $y$, dado que, en akabea, la negación de una cláusula verbal implica su nominalización y colocación en el slot argumental del sujeto de una cláusula copulativa (Zamponi y Comrie en prep: Secc. 5.2.), la estructura que esperamos ver en (25) debería contener un verbo copulativo plenamente expresado, como la cláusula copulativa en (26).

25. Éda [sic] yádí dutnga yábairé [sic]

$\begin{array}{llll}\varnothing \text {-oda yadi } & \text { dut-na } & \text { yaba } & \varnothing \text {-re } \\ 3-S & \text { esp._de_tortuga arponar-NOMZ NEG } & \text { RE-PRET } \\ \text { 'Ellos no arponaron ninguna tortuga comestible.' (lit. 'No hubo su } \\ \text { arponar tortugas'). (Portman 1887: 144) }\end{array}$

26. Kárin óda kóinga yábada

$\begin{array}{lll}\text { karin } \quad \varnothing \text {-oda } \quad \text { koi-na } & \text { yaba }=\text { da } \\ \text { aquí } 3-S \quad \text { danzar-NOMz } & \text { NEG }=\text { COP } \\ \text { 'Ellos no danzarán aquí.' (lit. 'No hay su danzar aquí'). } \\ \text { (Portman 1887: 108) }\end{array}$

En sostén de este posible tipo de elisión de la raíz verbal podemos citar otra oración registrada por Portman, reportada en (27), que puede, también, ser analizada como desprovista de la raíz copulativa eda $\sim=d a .{ }^{9}$

27.

... béringa-ke
berina $\quad \varnothing-k e \quad$ (o $\quad \begin{aligned} & \text { berina-ke) } \\ & \text { bueno } \quad \text { RE-nPSD }\end{aligned}$
'(El puerco) será bueno.' (Portman 1898: 119)

Un ulterior tipo de elisión de la raíz verbal se observa, con un mayor grado de seguridad, en una oración compleja opuchikwar contenida en el "manual" de Portman (véase ejemplo (28)). Este tipo de elisión establece que la raíz del verbo en la segunda de dos cláusulas yuxtapuestas puede ser omitida si la misma raíz es abiertamente expresada en la cláusula precedente y las dos partes de la oración son paralelas. En la oración opuchikwar en cuestión la yuxtaposición de las dos cláusulas que la componen sirve para expresar disyunción interrogativa.

28. Án ngóng chélewa [sic] lír írtilu [sic], án chélebírma kétewe [sic] liír? (opuchikwar)

$\begin{array}{lllll}\text { an } & \text { D-OD } & \text { telewa } & \text { I=ir-tilu } & \\ \text { S/N } & \text { 2-S.SG } & \text { buque } & \text { DEF=PS-ver } & \\ \text { an } & \text { tele-birma } & & \text { ketawa } & \text { I=ir- } \varnothing \\ \text { S/N } & \text { buque-chimenea } & \text { pequeño } & \text { DEF=PS-RE }\end{array}$

9 Varias raíces en akabea resultan ser bases lexicales multicategoriales. Específicamente, algunas raíces pueden presentarse tanto en función adjetival como verbal (Zamponi y Comrie en prep.: Secc. 3.1). No podemos excluir la posibilidad de que berina sea una de estas raíces. 
'¿Tú viste el buque o la lancha de vapor?' (lit. '¿Tú viste el buque? ¿Viste el buque de vapor pequeño?'). (Portman 1887: 126)

\section{Aspectos fonológicos y una analogía formal}

La elisión de la raíz verbal, en principio, puede conducir a problemas relacionados con la pronunciación del resultado, sobre todo en lenguas donde el acento cae o puede caer en la raíz, como en el caso del akabea, lengua en la que el acento ocurre siempre en la primera sílaba de aquella. Si el español tuviera el fenómeno de elisión de la raíz verbal, habría problemas en el caso de palabras como hablo (acento en la raíz), pero no en el caso de hablamos (acento en el sufijo temático) o hablaré (acento en el sufijo de persona y número). ¿Cómo se comportan las tres lenguas conocidas con elisión de la raíz verbal? En dos de estas lenguas la elisión de la raíz verbal parece no ocasionar problemas prosódicos, incluso de acento.

Respecto al inuktitut, según Swift y Allen (2002: 155), la estructura de la palabra fonológica está bien delimitada, aunque todavía no bien analizada. En general, coincide con la palabra ortográfica. En los ejemplos presentados por Swift y Allen las secuencias de sufijos que resultan de la elisión de la raíz verbal forman palabras ortográficas y, por presunción, fonológicas.

En kwaza, según van der Voort (2004: 579), hay tres principios muy importantes que regulan la posición del acento en una palabra fonológica: (a) cualquier morfema puede ser acentuado en el caso de énfasis o contraste; además, (b) algunos afijos atraen el acento, como el imperativo en $(9 b-c)$ y el negativo en (10b-c); además, (c) el acento cae en la primera sílaba de la palabra fonológica, como en $(1 b),(8 b)$ y $(11)$. De esa manera, una palabra fonológica que carece de raíz y consiste, por eso, en una serie de sufijos puede todavía recibir un acento según los principios generales.

En akabea y las demás lenguas gran andamanesas, la situación es más compleja. Las lenguas están muertas y no hay ninguna grabación sonora. La única evidencia consiste en las declaraciones explícitas de los autores sobre la posición del acento y su práctica respecto a la división en palabras ortográficas. La regla general en estas lenguas es que el acento cae en la primera sílaba de la raíz (Zamponi y Comrie en prep.: Secc. 2.4). Es evidente que esta regla no se puede aplicar a palabras que no tienen raíz. En el caso de formas verbales sin raíz que consisten exclusivamente en un sufijo, el principio seguido por los autores es unir ortográficamente el sufijo a la palabra precedente, como en los ejemplos (14)-(20) y (25), y esto nos permite suponer que el resultado es una única palabra fonológica. Si la forma verbal sin raíz tiene uno o más prefijos ( $y / 0$ eventualmente un proclítico), la secuencia forma una palabra ortográfica independiente, como en (21b), (22), (23a), (24) y (28), y plausiblemente también una palabra fonológica independiente. En este caso, no sabemos qué sílaba lleva el acento, ya que la forma no cae bajo la única regla dada por los autores, quienes no suelen marcar el acento en su transcripción.

Llamamos la atención al hecho de que raíces aparentemente elididas puedan también producirse por medio de una ciega operación de reglas 
fonológicas. Es evidente que ejemplos de este tipo no tienen nada que ver con el fenómeno de elisión de la raíz verbal que forma el tema de este artículo, guiado, como hemos visto, por principios discursivos.

En japonés (Martin 1988: 514-515, 538-539), la vocal inicial de los verbos auxiliares $i-r u$ e $i k-u$ puede ser suprimida después de una vocal. La interpretación literal de estos verbos fuera de construcciones auxiliares es, respectivamente, 'estar (de un ser animado)' e 'ir'. En su calidad de verbos auxiliares, $i$-ru expresa el aspecto progresivo o resultativo, sin restricción de animacidad en el sujeto, mientras que $i k-u$ tiene varias interpretaciones, incluso la realización gradual de una meta.

Ya que la raíz del verbo i-ru consiste en una única vocal, esta supresión vocálica conduce a una raíz cero, mientras que en el caso de $i k-u$ se conserva la consonante $k$, de manera que $k$ - constituye un alomorfo de $i k-$, como se puede ver en los ejemplos (29) y (30).

$\begin{array}{lll}\text { 29. a. } & \text { nat-te } & \text { ik-u } \\ & \text { nat-te } & k-u \\ \text { ponerse-cVB } & \text { ir-PRS } \\ & \text { 'se está aumentando gradualmente' } \\ \text { b. } & \text { ture-rare-te } & \text { it-ta } \\ & \text { ture-rare-te } & t-t a \\ & \text { llevar-PAS-CVB } \quad \text { ir-PRET } \\ & \text { 'estaba siendo llevado' }\end{array}$

$\begin{array}{lll}\text { 30. a. } & \text { si-te } & i \text {-ru } \\ & \text { si-te } & \emptyset \text {-ru } \\ & \text { hacer-CVB } & \text { estar-PRS } \\ & \text { 'está haciendo' } & \\ \text { b. } & \text { si-te } & \\ & \text { si-te } & \text { i-ta } \\ & \text { hacer-CVB } & \text {-ta } \\ & \text { 'estaba haciendo' } & \text { estar-PRET }\end{array}$

\section{Analogías funcionales y semánticas con los fenómenos de la elisión verbal y de las raíces cero}

La elisión de la raíz verbal muestra una clara analogía funcional con el fenómeno más común de la elisión verbal determinada por el contexto, es decir la omisión de una palabra verbal completa recuperable en el contexto lingüístico inmediatamente anterior. Específicamente es el tipo de elisión verbal intraoracional (o sintáctica: A -"¿Adónde vas?" B -"Al cine") al que la elisión de la raíz verbal se conecta. Como hemos visto en las páginas anteriores, en inuktitut y kwaza, como en akabea, la elisión de la raíz verbal es comúnmente permitida cuando la forma completa de un verbo puede ser deducida del material lingüístico precedente y se manifiesta, típicamente, en 
intercambios dialógicos, con la supresión de un elemento que, de otro modo, se repetiría de un turno conversacional anterior.

Inuktitut: "Elliptical constructions are often used in contiguous utterance pairs, such as a request and elliptical response or a question and elliptical answer" (Swift and Allen 2002: 135).

31. a. Anaana qajurturumajunga

anaana qajuq-tuq-guma-junga

madre sopa-consumir-querer-PAR.1SGS

'Madre, quiero tomar una sopa.'

b. Nialirqutit siaru

$\begin{array}{ll}\varnothing \text {-niaq-liq-vutit } & \text { siaru } \\ \text { BE-HOY-FUT-ING-IND.2sGS } & \text { después }\end{array}$

'[Tomarás la sopa] después.' (Swift and Allen 2002: 136)

Kwaza: "...root ellipsis occur frequently in dialogues, as a minimal response to questions, remarks or comments". "About elliptic responses ..., one could say that the specific root is physically absent but that it is understood, and identifiable from the context" (ibid., p. 588).

32
a. tsũhũ-ra'ti
e-e'hỹ-wa-re
qué-FOC
RED-hacer-SI-INT
'¿Qué están haciendo?'
b. $\varnothing$-tsy-'re
RE-GER-INT
'No sé [qué están haciendo].' (van der Voort 2004: 580)

Akabea: "In all cases of this kind [of elliptical use of verbs] the actual sense of these verbs is derived from the circumstances under which the sentences where they occur are spoken. ... The use of such elliptical sentences as these shows up the intensely colloquial character of the language in a strong light" (Man 1878: Ch. 34). Véase los ejemplos (13) y (14) en sección 2.3.

En akabea y, por lo menos, en otras dos lenguas gran andamanesas (opuchikwar y okojuwoi) hay también un tipo de elisión de la raíz verbal que podríamos definir intraoracional e incluso, más específicamente, intraclausal ya que no está condicionado por el contexto lingüístico precedente sino, más bien, por la semántica de una categoría léxica particular presente en la cláusula en la que la elisión ocurre. Los verbos implicados en este tipo de elisión incluyen 'venir, ir' y algunas formas con el significado básico de 'golpear (con un palo o un arma)' dotadas de un prefijo somático que especifica la parte del cuerpo golpeada.

La categoría léxica a la cual podemos reconocer el específico rol de "licenciador" (licensor) de la elisión de la raíz verbal 'venir, ir' es un constituyente (un adverbio o un sintagma posposicional cualquiera sea) que 
aporta una especificación del destino del movimiento: lo que podríamos llamar, en inglés, un "MOTION-GOAL licensor". Esto se deduce de los ejemplos (15)(17) en 2.3. En (16) hay una ulterior categoría que puede o no tener un rol en permitir la elisión de la raíz verbal: el sujeto do 'yo', es decir el "MOVER". El MOVER no puede ser considerado un efectivo licenciador ya que las oraciones (15) y (17), en las cuales no figura, están gramaticalmente bien formadas. Pero es verosímil creer que el pronombre do en (16) facilite, de alguna manera, la elisión de la raíz verbal sin ser necesariamente requerido. En (18) el MOVER está expresado abiertamente, pero no hay un MOTION-GOAL licensor. Esto indica que tampoco el GOAL, en realidad, es un verdadero licenciador de la raíz on 'venir, ir'. Nuestra impresión, sin embargo, es que la oración en (18) es una expresión idiomática. En nuestra opinión, lo que estamos tratando, en otras palabras, es simplemente un caso de elisión de la raíz verbal convencionalizada.

La elisión de la raíz 'golpear (con un palo o un arma)' requiere que un PACIENTE sea abiertamente expresado (véase los ejemplos (21b-24) en 2.3). Quien golpea, el "HITTER", no es un licenciador, dado que no viene indicado en (24). Probablemente, su expresión en (21b) y (22) facilita, sin embargo, la elisión. PACIENTE y HITTER son realizados por simples pronombres en ( $21 b$ 23c), excluido el ejemplo (22) en el cual el HITTER está expresado por un sintagma nominal compuesto por un pronombre demostrativo y un sustantivo. El HITTER puede ser un ser humano $(21 b)$ o un instrumento (22). EI PACIENTE es siempre un ser humano en $(21 b-24)$.

Si nuestra interpretación de los ejemplos (25) y (27) como construcciones elípticas sin raíz copulativa eda $\sim=d a$ es correcta, el constituyente sujeto en una cláusula copulativa existencial (25) y el complemento en una cláusula copulativa nominal (27) son ulteriores licenciadores de la elisión de la raíz intraclausal.

Por el hecho de depender de un específico licenciador, el tipo de elisión de la raíz verbal intraclausal que se observa en akabea evoca la estrategia de elisión verbal intraoracional que McShane (2005: 154) define "Multilicensor Verbal Ellipsis", aunque esta establezca, como el término indica, que sea la semántica combinada de más (al menos dos) categorías abiertas a legitimar la elisión y, a veces, con la ayuda del contexto, a asegurar una recuperabilidad completa del significado verbal.

Es interesante notar que las raíces verbales sujetas en akabea a elisión intraclausal expresan el mismo significado léxico de los verbos envueltos en la "Multilicensor Verbal Ellipsis" en otras lenguas, como muestra el cuadro $1 .{ }^{10}$

\footnotetext{
${ }^{10}$ Es un hecho bien notorio que la mayor parte de las lenguas germánicas pueden usar los verbos modales con complementos no verbales (direccionales) sin necesidad de un verbo de movimiento; cf. el alemán Ich muss nach Leipzig (gehen) 'Tengo que (ir) a Leipzig' y el neerlandés Jan wil weg (gaan) 'Juan quiere (irse)'. Han sido propuestas dos maneras de analizar tales construcciones. La opinión predominante es que los verbos modales, en casos como estos, son verbos principales, es decir verbos plenamente léxicos. Según otra interpretación, los modales son verbos funcionales, es decir auxiliares, y, en la estructura sintáctica, hay un verbo de movimiento silente (Geerts et al. 1984: 558, Vanden Wyngaerd 1994: 65-68, van Riemsdijk 2002, 2012). Otra interpretación posible hace referencia al fenómeno de la elisión verbal: en lugar de la inserción léxica de una forma nula, entraría en juego un completo borramiento del verbo de movimiento ('ir'). Esta opción, sin embargo, fue rechazada por Barbiers (1995: 150-54) con un buen número de argumentos.
} 
Cuadro 1. Ejemplos de verbos sujetos a "Multilicensor Verbal Ellipsis" en diferentes lenguas

\begin{tabular}{|c|c|c|c|c|c|}
\hline Verbo(s) & Licenciadores & Lengua(s) & Familia & País(es) & Referencias \\
\hline \multirow[t]{4}{*}{$\begin{array}{l}\text { verbos de } \\
\text { movimiento }\end{array}$} & \multirow[t]{4}{*}{$\begin{array}{l}\text { MOVER + } \\
\text { MOTION-GOAL }\end{array}$} & ruso [rus] & indoeuropeo & Rusia & $\begin{array}{l}\text { McShane } \\
\text { (2005: 159-62) }\end{array}$ \\
\hline & & polaco $[\mathrm{pol}]$ & indoeuropeo & Polonia & $\begin{array}{l}\text { McShane } \\
(2005: 175)\end{array}$ \\
\hline & & $\begin{array}{l}\text { karo batak } \\
{[\text { bto] }}\end{array}$ & austronesio & Indonesia & $\begin{array}{l}\text { Woollam } \\
\text { (1996: 289) }\end{array}$ \\
\hline & & gayo [gay] & austronesio & Indonesia & $\begin{array}{l}\text { Eades } \\
(2005: 140)\end{array}$ \\
\hline 'veni r' & $\begin{array}{l}\text { MOVER + } \\
\text { PARTÍCULA }\end{array}$ & $\begin{array}{l}\text { toqabaqi ta } \\
\text { [mlu] }\end{array}$ & austronesio & $\begin{array}{l}\text { Islas } \\
\text { Sal omón }\end{array}$ & $\begin{array}{l}\text { Lichtenberk } \\
\text { (2008: 945) }\end{array}$ \\
\hline \multirow[t]{3}{*}{ 'golpear' } & \multirow{3}{*}{$\begin{array}{l}\text { HITTER + } \\
\text { PACIENTE + } \\
\text { INSTRUMENTO } \\
\text { y/o SITIO }\end{array}$} & ruso [rus] & indoeuropeo & Rusia & $\begin{array}{l}\text { McShane } \\
(2005: 158)\end{array}$ \\
\hline & & polaco [pol] & indoeuropeo & Pol onia & $\begin{array}{l}\text { McShane } \\
\text { (2005: 173) }\end{array}$ \\
\hline & & checo [ces] & indoeuropeo & $\begin{array}{l}\text { República } \\
\text { Checa }\end{array}$ & $\begin{array}{l}\text { McShane } \\
(2005: 173)\end{array}$ \\
\hline \multirow[t]{4}{*}{ 'ser' } & \multirow[t]{4}{*}{$\begin{array}{l}\text { SUJETO + } \\
\text { COMPLEMENTO }^{b}\end{array}$} & $\begin{array}{l}\text { cantonés } \\
\text { [yue] }\end{array}$ & sino-tibetano & China & $\begin{array}{l}\text { Matthews y Yip } \\
(2011: 145)\end{array}$ \\
\hline & & kam [kmc] & tai-kadai & China & $\begin{array}{l}\text { Yang y } \\
\text { Edmondson } \\
\text { (2008: 532) }\end{array}$ \\
\hline & & $\begin{array}{l}\text { mohave } \\
\text { [mov] }\end{array}$ & \begin{tabular}{|l} 
yumano- \\
cochimí
\end{tabular} & $\begin{array}{l}\text { Estados } \\
\text { Unidos }\end{array}$ & $\begin{array}{l}\text { Munro } \\
(1976: 50)\end{array}$ \\
\hline & & $\begin{array}{l}\text { cavineña } \\
\text { [cav] }\end{array}$ & tacana(no) & Bolivia & $\begin{array}{l}\text { Guillaume } \\
\text { (2008: 97) }\end{array}$ \\
\hline
\end{tabular}

${ }^{a}$ Y, también, MOTION-GOAL y varios otros licenciadores en ruso.

${ }^{\mathrm{b}}$ En ausencia de particulares condiciones gramaticales.

Se observen ahora algunos ejemplos concretos de "Multilicensor Verbal Ellipsis".
33. $a k u$
ben
ari
kedé
yo
recién
de
mercado
'Yo recién [vine] del mercado.' (Eades 2005: 140)
34. Ja
emu
ne
sil'no
Yo:NOM
él.DAT
NEG
fuerte
(ruso)
'Yo no lo [golpeé] fuerte.' (McShane 2005: 158)
$\begin{array}{llllll}\text { 35. } & \text { gó } & \text { bun } & \text { syū } & \text { ngóh } & \text { ge } \\ \text { aquel } & \mathrm{CL} & \text { libro } & \text { yo } & \mathrm{PC} \\ \text { 'Aquel libro } & \text { [es] } & \text { mío.' (Matthews y Yip } & 2011: & 145 \text { ) }\end{array}$

Es igualmente interesante notar que los mismos significados de las raíces verbales sujetas en akabea a elisión intraclausal, en algunas otras lenguas, son expresados por raíces verbales que tienen un alomorfo desprovisto de forma fónica (véase 'estar en movimiento' en madi (dialecto jarawara), lengua arawá de Brasil (ejemplo (36)) y el cuadro 2 para una lista de otros casos señalados en la literatura) o por raíces que quedan siempre silentes, como en el caso de 
'moverse a pie' en el nimboran [nir] (familia nimboranica) de Papúa Occidental, en formas que, sin embargo, incluyen obligatoriamente un sufijo direccional que implica movimiento (May 1997: 105-106) (37), y de 'golpear, matar' en el bukiyip [ape] (familia torricelina nuclear) (38) y en el selepet [spl] (trans-neoguineano nuclear) (39) de Papúa Nueva Guinea.

36. a. bati ka-ke

padre estar_en_movimiento-VINIENDO

'El padre está viniendo.'

(madi [jarawara])

b. $o-\varnothing-k e$

1SGS-estar_en_movimiento-VINIENDO

'Estoy viniendo.' (Dixon 2009: 127)

37. Ø-ke-ban-t-e

moverse_a_pie-DUS-DESDE_ABAJO_A_AQUÍ-PRS-2S

(nimboran)

'Ya viniste aquí arriba.' (May 1997: 106).

38. nabotik ch-a- Ø-nú n-a-gak

ayer 3PL.MIXS-REAL-golpear-3SG.MO 3SG.MS-REAL-morir

'Ayer lo golpearon y él se murió.' (Conrad y Wogiga 1991: 18)

39. Ø-noho-ap

(selepet)

golpear-1SGO-3SGS.PSDINM

'Él me golpeó.' (McElhanon 1973: 43)

Cuadro 2. Ejemplos de raíces verbales con un alomorfo sin forma fónica en diferentes lenguas.

\begin{tabular}{|c|c|c|c|c|}
\hline Significado(s) & Lengua(s) & Familia & País(es) & Referencias \\
\hline $\begin{array}{l}\text { 'estar en } \\
\text { movimiento' }\end{array}$ & udí [udi $]^{\mathrm{a}}$ & $\begin{array}{l}\text { najsko- } \\
\text { daguestano }\end{array}$ & $\begin{array}{l}\text { Azerbaiyán, } \\
\text { Rusia, Georgia }\end{array}$ & $\begin{array}{l}\text { Harris } \\
(2008: 220)\end{array}$ \\
\hline 'venir', 'ir' & 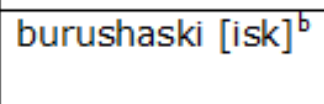 & lengua aisl ada & Pakistán & $\begin{array}{l}\text { Tikkanen } \\
\text { (1999: 297) }\end{array}$ \\
\hline 'golpear, matar' & kâte [kmg] & $\begin{array}{l}\text { trans-neoguineano } \\
\text { nuclear }\end{array}$ & $\begin{array}{l}\text { Papúa } \\
\text { Nueva Guinea }\end{array}$ & $\begin{array}{l}\text { Suter } \\
(2014: 19)\end{array}$ \\
\hline \multirow[t]{4}{*}{ 'ser' } & vasco [eus] & Iengua aislada & $\begin{array}{l}\text { España, } \\
\text { Francia }\end{array}$ & $\begin{array}{l}\text { Saltarelli } \\
\text { (1988: } 302)\end{array}$ \\
\hline & itelmeno [itl] & chucoto-camchatco & Rusia & $\begin{array}{l}\text { Holst } \\
\text { (2014: } 39)\end{array}$ \\
\hline & wai wai [waw] & caribe & Guyana, Brasil & $\begin{array}{l}\text { Hawkins } \\
(1998: 167-168)\end{array}$ \\
\hline & kulina [cul] & arawá & Brasil, Perú & $\begin{array}{l}\text { Dienst } \\
\text { (2014: 233) }\end{array}$ \\
\hline
\end{tabular}

a El alomorfo sin forma fónica ocurre con preverbos locativos (con los cuales expresa significados como 'venir', 'ir', 'ir arriba' e 'ir abajo') en formas del presente.

b En el paradigma converbal, los dos verbos 'venir' e 'ir' se distinguen solo por los prefijos $d$ (que ocurre en 'venir' y es un morfema derivacional con un significado opaco) y $n$ - (que ocurre en 'ir' y es, primariamente, un morfema converbal), como en $d$-áa- $\varnothing$ - $n$ 'habiendo yo venido' and $n$-áa- $\varnothing-n$ 'habiendo yo ido'. 
Podríamos concluir ahora que la elisión de la raíz 'venir, ir' en akabea se alinea con una tendencia de los conceptos verbales de movimiento a recibir una expresión lingüística máximamente reducida, especialmente en formas que presentan afijos que inherentemente aportan una indicación de movimiento (nimboran y udí), o a no ser de modo alguno expresados en presencia de un constituyente en la oración que proporciona la misma especificación (ruso, polaco, karo batak, gayo y toqabaqita).

El verbo 'golpear' comparte con los verbos de movimiento los matices de velocidad e inmediatez. "Golpear" es probablemente la más simple acción intencional que un agente puede ejecutar sobre un paciente. La expresión lingüística (a veces) faltante de esta acción (selepet, bukiyip y kâte) y la completa omisibilidad del verbo o raíz verbal que la expresa, posible en presencia de un PACIENTE abiertamente expresado $y$, por lo menos, facilitada por la presencia de un HITTER manifiesto (akabea, ruso, polaco y checo), en nuestra opinión, deben necesariamente estar vinculados al carácter cognitivamente básico de la misma acción.

Por lo que concierne el verbo copulativo 'ser', que carece completamente de significado referencial, la eventual elisión de su raíz en akabea se encuadra perfectamente en el carácter ancilar del verbo en cuestión que justifica también su elisión obligatoria u opcional en muchas lenguas del mundo, sobre todo en las formas menos marcadas del punto de vista temporal (como el tiempo presente o el aoristo; Wetzer 1996: 134), y la presencia, en algunas lenguas de raíces verbales copulativas que no tienen expresión lingüística en determinadas formas (como en vasco, itelmeno, wai wai y kulina).

El ulterior tipo de elisión de la raíz verbal intraoracional que se observa exclusivamente en una oración opuchikwar consistente en dos cláusulas yuxtapuestas (ejemplo (29) en 2.3) puede ser definido como interclausal. En la oración en cuestión, en lugar de una raíz verbal que sería idéntica a la raíz del primer verbo, en la primera cláusula, se observa la completa ausencia de un morfema indicado por forma fónica.

Este tipo de elisión de la raíz verbal aparece, por tanto, paralelo a la estrategia de elisión verbal intraoracional conocida como Vaciado o Gapping, que permite la no expresión del verbo y, opcionalmente, de otros elementos del sintagma verbal de la(s) cláusula(s) no inicial(es) de una estructura coordinada (Gabriela telefoneó a Carlos y Lucía $\varnothing$ a Daniel) o comparativa (Graciela ama a su gato como una madre $\varnothing$ a su hijo).

\section{Conclusiones}

Esperamos haber mostrado que la elisión de la raíz verbal es un fenómeno inequívocamente atestiguado, aunque tipológicamente raro, que consiste en la supresión de la raíz de un verbo, en primer lugar bajo condiciones discursivas, y que se encuentra en las lenguas inuktitut y kwaza y, por lo menos, en algunos miembros de la familia gran andamanesa.

Generalmente, la elisión de la raíz verbal ocurre cuando el contenido de la raíz elidida es recuperable desde el contexto lingüístico o extralingüístico, aunque se encuentren también, en lenguas específicas, casos de elisión de 
raíces de verbos muy básicos, tales como el verbo intransitivo 'ir, venir' y el verbo transitivo 'golpear'.

La elisión de la raíz verbal tiene paralelos formales con las raíces (y alomorfos de raíces) cero, ya que, en ambos casos, hay una raíz sin expresión fonética. Los dos fenómenos se distinguen sin embargo por cuanto la raíz cero es un fenómeno léxico (algunas unidades léxicas tienen una raíz o un alomorfo cero), mientras que la elisión de la raíz verbal es ante todo un fenómeno discursivo.

Hay también paralelos con la elisión del verbo (es decir de la palabra entera), sobre todo en condiciones discursivas que regulan ambas posibilidades, aunque la elisión de una palabra entera no conduce a los problemas morfológicos y fonológicos ocasionados por la elisión de la raíz verbal.

\section{Abreviaturas}

$\begin{array}{llll}\text { ABS } & \text { absolutivo } & \text { nPSD } & \text { no pasado } \\ \text { AL } & \text { alativo } & \text { O } & \text { objeto } \\ \text { BE } & \text { base elidida } & \text { PA } & \text { persona asociada } \\ \text { CL } & \text { clasificador } & \text { PAR } & \text { participial } \\ \text { COP } & \text { cópula } & \text { PAS } & \text { pasivo } \\ \text { COR } & \text { cortés } & \text { PC } & \text { partícula de conexión } \\ \text { CTG } & \text { contingente } & \text { PL } & \text { plural } \\ \text { CVB } & \text { converbo } & \text { PLAS } & \text { plural asociativo } \\ \text { DAT } & \text { dativo } & \text { POSP } & \text { posponedor (procrastinative) } \\ \text { DEC } & \text { declarativo } & \text { POT } & \text { potencial } \\ \text { DEM } & \text { demostrativo } & \text { PRET } & \text { pretérito } \\ \text { DIST } & \text { distante } & \text { PREV } & \text { preverbo } \\ \text { DU } & \text { dual } & \text { PROX } & \text { proximal } \\ \text { DUB } & \text { dubitativo } & \text { PRS } & \text { presente } \\ \text { ENF } & \text { enfático } & \text { PS } & \text { prefijo somático } \\ \text { ESP } & \text { espacial } & \text { PSDINM } & \text { pasado inmediato } \\ \text { FOC } & \text { focalizador } & \text { RE } & \text { raíz elidida } \\ \text { FUT } & \text { futuro } & \text { RED } & \text { reduplicación } \\ \text { FUTPROX } & \text { futuro próximo } & \text { REAL } & \text { realis } \\ \text { GER } & \text { gerundio } & \text { REFL } & \text { reflexivo } \\ \text { IMP } & \text { imperativo } & \text { SG } & \text { singular } \\ \text { IND } & \text { indicativo } & \text { S } & \text { sujeto } \\ \text { ING } & \text { ingresivo } & \text { SI } & \text { sujeto indefinido } \\ \text { INT } & \text { interrogativo } & \text { TAM } & \text { tiempo-aspecto-modo } \\ \text { LOC } & \text { locativo } & \text { TERM } & \text { terminativo } \\ \text { M } & \text { masculino } & \text { VOL } & \text { volitivo } \\ \text { MIX } & \text { (género) mixto } & & \\ \text { NEG } & \text { negativo } & & \\ \text { NOMZ } & \text { nominalizador } & & \\ \text { NOM } & \text { nominativo } & & \end{array}$


$X>$ Ysujeto/agente $X$ que actúa sobre el objeto/paciente $Y$ o posesor $X$ del poseído $Y$

\section{Referencias bibliográficas}

Abbi, Anvita. 2012. Dictionary of the Great Andamanese language: EnglishGreat Andamanese-Hindi, Delhi, Ratna Sagar.

Abbi, Anvita. 2013. A grammar of the Great Andamanese language: an ethnolinguistic study, Leiden/Boston, Brill.

Barbiers, Sjef. 1995. The syntax of interpretations, The Hague, Holland Academic Graphic.

Comrie, Bernard y Raoul Zamponi. En prep. Verb root ellipsis. Ms.

Conrad, Robert J. y Kepas Wogiga, 1991. An outline of Bukiyip grammar, Canberra, The Australian National University.

Dienst, Stefan, 2014. A grammar of Kulina, Berlin/Boston, De Gruyter Mouton.

Dixon, R. M. W. 2009. Zero and nothing in Jarawara, en Johannes Helmbrecht et al. (eds.), Form and function in language research: papers in honour of Christian Lehmann, Berlin/New York, Mouton de Gruyter: 125-137.

Eades, Domenyk, 2005. A grammar of Gayo: a language of Aceh, Sumatra, Canberra, The Australian National University.

Geerts, G., W. Haeseryn, J. de Rooij y M. C. van den Toorn. 1984. Algemene Nederlandse spraakkunst, Groningen, Wolters-Noordhoff.

Guillaume, Antoine. 2008. A grammar of Cavineña, Berlin/New York, Mouton de Gruyter.

Harris, Alice C. 2008. Light verbs as classifiers in Udi, Diachronica, 25: 213241.

Hawkins, Robert E., 1998. Wai Wai, en Desmond C. Derbyshire y Geoffrey K. Pullum (eds.), Handbook of Amazonian languages Vol. 4, Berlin/New York, Mouton de Gruyter: 2-224.

Holst, Jan Henrik, 2014. Advances in Burushaski linguistics, Tubingen, Narr.

Lichtenberk, Frantisek. 2008. A grammar of Toqabaqita, Berlin/New York, Mouton de Gruyter.

Man, Edward H. 1878. Andamanese grammar, annotated by Sir R. C. Temple. Ms. no. 111 en la colección Edward H. Man del Royal Anthropological Institute of Great Britain and Ireland, London.

Man, Edward H. 1919-1923. A dictionary of the South Andaman (Âkà-Bêa) language, Indian Antiquary 48 (1919) Supl. 1-84, 49 (1920) Supl. 85136, 50 (1921) Supl. 137-164, 51 (1922) Supl. 165-188, 52 (1923) Supl.: 189-203.

Martin, Samuel E. 1988. A reference grammar of Japanese (2da edición), Rutland, VT/Tokyo: Charles E. Tuttle.

Matthews, Stephen y Virginia Yip. 2011. Cantonese: a comprehensive grammar (2da edición), London/New York, Routledge.

May, Kevin. 1997. A study of the Nimboran language: phonology, morphology, and phrase structure, Tesis de Maestría, La Trobe University, Bundoora. (https://books.google.com.uy/books?id=4YUeNQAACAAJ\&dq=inauthor: \% 22Kevin+May\%22\&hl=es-) 
McElhanon, Kenneth A. 1973. Towards a typology of the Finisterre-Huon languages, New Guinea, Canberra, The Australian National University.

McShane, Marjorie J. 2005. A theory of ellipsis, Oxford, Oxford University Press.

Munro, Pamela. 1976. Mojave syntax, New York/London, Garland.

Portman, Maurice V. 1887. Manual of the Andamanese languages, London, W. H. Allen.

Portman, Maurice V. 1898. Notes on the languages of the South Andaman group of tribes, Calcutta, Office of the Superintendent of Government Printing.

Radcliffe-Brown, Alfred R. 1933. The Andaman islanders, 2da ed., Cambridge, The University Press.

Saltarelli, Mario. 1988. Basque, London/New York, Routledge.

Suter, Edgar. 2014. Kâte he 'hit' and qa 'hit': a study in lexicology, Language \& Linguistics in Melanesia, 32: 18-57.

Swift, Mary D. y Shanley E. M. Allen. 2002. Verb base ellipsis in Inuktitut conversational discourse, International Journal of American Linguistics, 68: $133-156$.

Tikkanen, Bertil. 1999. Concerning the typology of Burushaski and the roots of its prefixes $d$ - and $n$-. Studia Orientalia, 85: 277-300.

Trommer, Jochen. 2012. Ø-exponence, en Jochen Trommer (ed.), The morphology and phonology of exponence, Oxford, Oxford University Press: $326-354$.

van der Voort, Hein. 2004. A grammar of Kwaza, Berlin/New York, Mouton de Gruyter.

van Riemsdijk, Henk C. 2002. The unbearable lightness of GOing. The projection parameter as a pure parameter governing the distribution of elliptic motion verbs in Germanic, Journal of Comparative Germanic Linguistics, 5: 143-196.

van Riemsdijk, Henk C. 2012. The absent, the silent, and the audible: some thoughts on the morphology of silent verbs, en Esther Torrego (ed.), Of grammar, words, and verses: in honor of Carlos Piera, Amsterdam, John Benjamins: 19-39.

Vanden Wyngaerd, Guido. 1994. PRO-legomena: distribution and reference of infinitival subjects, Berlin/New York, Mouton de Gruyter.

Wetzer, Harrie. 1996. The typology of adjectival predication, Berlin/New York, Mouton de Gruyter.

Woollam, Wilfred. 1996. A grammar of Karo Batak, Sumatra, Canberra, The Australian National University.

Yang Tongyin y Jerold A. Edmondson. 2008. Kam, en Anthony V. N. Diller, Jerold A. Edmondson y Luo Yongxian (eds.), The Tai-Kadai languages, London/New York, Routledge: 509-584.

Zamponi, Raoul y Bernard Comrie. En prep. A grammar of Akabea. Ms. 